\title{
Herzbeuteltamponade beim Hund - diagnostisches Vorgehen und Therapie
}

\author{
Katrin Baldauf
}

Die meisten Patienten mit einem Perikarderguss können durch eine gründliche klinische Untersuchung erkannt werden. Die Befunde umfassen gedämpfte Herztöne, paradoxen Puls und gestaute Jugularvenen mit und ohne Aszites. Eine ätiologische Diagnose ist anhand der Ergussanalyse nur selten möglich und die Prognose variiert deutlich je nach Ursache des Ergusses.

\section{Der Patient}

\section{Signalement}

Am häufigsten betroffen sind ältere Hunde größerer Rassen. Es wird ein mittleres Alter von 9,7 \pm 2 ,2 Jahre bei einem mittleren Gewicht von 31,2 $\pm 12,6 \mathrm{~kg}$ beschrieben [44]. Dabei ist keine Geschlechtsprädisposition erkennbar. Golden Retriever sind überrepräsentiert [44], gefolgt von Deutschen Schäferhunden. Mit etwas Abstand folgen Bernhardiner, Neufundländer, Labrador Retriever und Mischlinge [42].

\section{Vorstellungsgrund}

Die Anamnese unterscheidet sich deutlich je nachdem, ob es sich um einen akuten oder chronischen Erguss handelt.

Bei einem chronischen Perikarderguss steht klinisch eine kongestive Rechtsherzinsuffizienz im Vordergrund, die für die Besitzer insbesondere durch ein umfangsvermehrtes Abdomen, Lethargie, Belastungsintoleranz, Kurzatmigkeit und reduzierten Appetit auffällig wird.

Bei einem akuten Perikarderguss überwiegen Symptome eines verringerten Auswurfs, die sich insbesondere nach körperlicher Belastung in Schwäche und Kollaps äußern [41]. Weitere mögliche Krankheitszeichen sind Polydipsie, Erbrechen und Husten [44].

\section{Klinisches Bild}

Die Kardinalsymptome können historisch als Beck-Trias zusammengefasst werden [44]:

- gestaute Jugularvenen

- schwacher Femoralispuls

- gedämpfte Herztöne

Die Erklärung für die Befunde liegt in der intrakardialen Druckverteilung.

\section{Gestaute Jugularvenen}

Da physiologisch im rechten Atrium und rechten Ventrikel im Vergleich zur linken Herzseite der geringere Druck herrscht, wird bei Erreichen dieses Druckes die Füllung insbesondere der rechten Herzseite behindert. Die klinische Konsequenz ist ein erhöhter rechtsatrialer Füllungsdruck, der sich in einer venösen Stauung, erkennbar an erweiterten Jugularvenen ( $\triangleright$ Abb. 1) und bei längerem Bestehen an einem Aszites, manifestiert. Für die Untersuchung der Jugularvenen kann Scheren oder zumindest das Befeuchten und Scheiteln des Felles erforderlich sein.

\section{konkret}

Alle Hunde mit Herzbeuteltamponade haben gestaute Jugularvenen [37].

\section{Schwacher Femoralispuls}

Durch die verminderte Aufnahme von Blut kann die rechte Herzseite weniger Blut über den Lungenkreislauf an das linke Herz liefern. Infolgedessen wird weniger Blut in den systemischen Kreislauf gebracht. Somit wird die Pulsqualität schlechter und das Herz reagiert mit einer gesteigerten Herzfrequenz, um das Herzminutenvolumen aufrechtzuhalten.

\section{Gedämpfte Herztöne}

Eine akustische Dämpfung kann durch die Flüssigkeit im Herzbeutel erfolgen. Allerdings kann insbesondere bei akuten Ergüssen bereits eine geringe Ergussmenge für eine Tamponade ausreichen, sodass dieser Befund nicht vorliegen muss [37].

\section{Paradoxer Puls}

Ein stark hinweisender Befund ist der paradoxe Puls. Ein gesundes Herz adaptiert das Auswurfvolumen automatisch an die jeweiligen intrathorakalen Druckverhältnisse. Somit gibt es physiologischerweise keine Unterschiede der Pulsqualität bei In- und Exspiration. Wenn bei Exspiration ein kräftigerer Puls als bei Inspiration auftritt, ist dieser Befund stark hinweisend auf einen Perikarderguss (Differenzialdiagnosen: andere restriktive Perikarderkrankungen oder Spannungspneumothorax) [37].

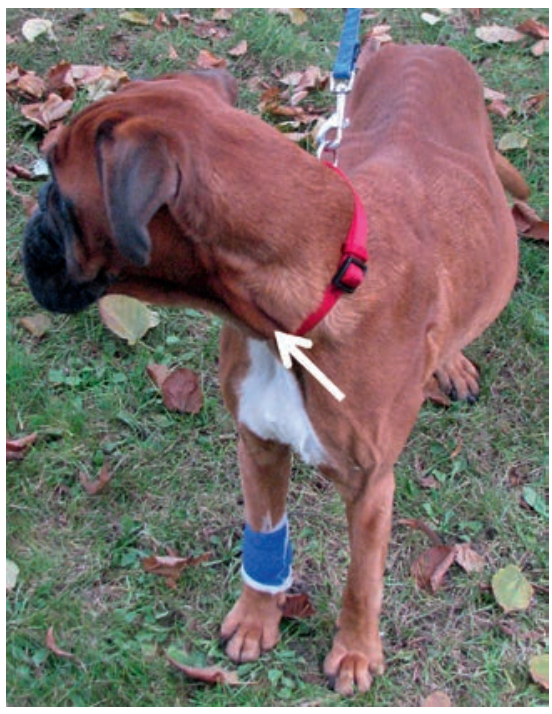

Abb. 1 Boxer, 4 Jahre, weiblich. Kongestive Rechtsherzinsuffizienz mit hochgradig gestauten Jugularvenen (Pfeil) und Abmagerung anderer Ursache. 
Weitere Befunde können Tachykardie, Hepatomegalie, Aszites, blasse Schleimhäute und Tachy- oder Dyspnoe sein [27].

\section{Diagnostik}

\section{Blutuntersuchungen}

Eine milde Anämie kann durch den Blutverlust oder durch eine chronische Erkrankung auftreten. Auch eine dezente Leukozytose wird häufig beobachtet [4]. Kernhaltige Erythrozyten, Schistozyten oder Akanthozyten können insbesondere bei einem Hämangiosarkom festzustellen sein [37].

Die blutchemischen Parameter sind meistens nicht verändert. Eine prärenale Azotämie kann durch den verringerten Auswurf vorliegen. Erhöhte Leberenzymaktivitäten sind durch eine Stauung möglich. Auch eine milde Hyponatriämie, Hypochloridämie und Hyperkaliämie durch das verringerte zirkulierende Blutvolumen können insbesondere bei Hunden mit ausgeprägtem Aszites nachzuweisen sein [37].

Wenn eine extrakardiale Ursache (z.B. urämische Perikarditis [36], Koagulopathie [31,33], eventuell Hypothyreose [28]) vorliegt, kann diese in entsprechenden Blutuntersuchungen erkannt werden. Coccidioides immitis sollte bei Hunden aus endemischen Gebieten (z.B.
Texas) als mögliche Ursache bedacht werden [22].

Troponin I kann Hinweise auf das Vorliegen eines Hämangiosarkoms liefern [38]. Ein Wert von $>0,25 \mathrm{ng} / \mathrm{ml}$ weist bei Patienten mit Perikarderguss eine Sensitivität von $81 \%$ und eine Spezifität von $100 \%$ für das Vorliegen eines Hämangiosarkoms auf [7].

\section{Röntgenuntersuchung}

Eine gering- bis hochgradig vergrößerte Herzsilhouette kann in der Röntgenuntersuchung festgestellt werden [20]. Mit länger bestehendem Erguss können die Volumina des Ergusses zunehmen und massive Vergrößerungen ergeben. Die Herzsilhouette wirkt häufig abgerundet bis globoid („Fußballherz“). Der dorsokaudale Anteil der Herzsilhouette kann konvex erscheinen. Die Berandung des Herzens kann scharf konturiert erscheinen, da die tatsächliche Herzbewegung innerhalb des nicht kontraktilen, flüssigkeitsgefüllten Perikards stattfindet ( $\bullet$ Abb. 2 ).

Insgesamt sind die radiologischen Veränderungen der Herzsilhouette aber weder hochspezifisch noch hochsensitiv (>90\%) für den Nachweis eines tamponierenden Perikardergusses [9]. Insbesondere bei akut auftretenden Ergüssen reichen kleinere Volumina für eine Tamponade, bei der die Röntgenaufnahme noch normal aussehen kann [19].
Die V. cava caudalis kann verbreitert sein [29]. Als Orientierung dient der Vergleich zur Aorta (>1,5), zu dem thorakalen Wirbel über der Bifurkation der Trachea $(>1,3)$ oder zur 4 . Rippe $(>3,5)$ [26] ( Abb. 2).

Das Lungenparenchym sollte bei Patienten mit Perikarderguss weniger perfundiert sein und entsprechend der Pathophysiologie keine Hinweise auf eine kongestive Linksherzinsuffizienz aufweisen. Ein Pleuraerguss kann vorliegen.

Bei manchen Hunden sind Anzeichen von Metastasen festzustellen. Die Häufigkeit von Metastasen verschiedener neoplastischer Erkrankungen (Hämangiosarkom, Mesotheliom, Chemodektom, ektopes Schilddrüsenadenokarzinom) wird mit bis zu 50-66\% angegeben [27].

\section{Elektrokardiogramm}

Eine Sinustachykardie ist ein häufiger Befund [37]. Seltener können ventrikuläre Extrasystolen ( $\triangleright$ Abb.3) festzustellen sein. Diese treten häufiger nach der Perikardiozentese auf. Eine Niedervoltage (QRS-Komplexe $<1 \mathrm{mV}$ in allen Ableitungen) kann bei ca. 50\% der Hunde wegen des erhöhten elektrischen Widerstands der Ergussflüssigkeit vorliegen [4].

Ein elektrischer Alternans (variierende Höhe der R-Zacken) ist, wenn vorhanden, ein typischer Befund. Dieser Befund entsteht durch ein „Schwimmen“ des Her-

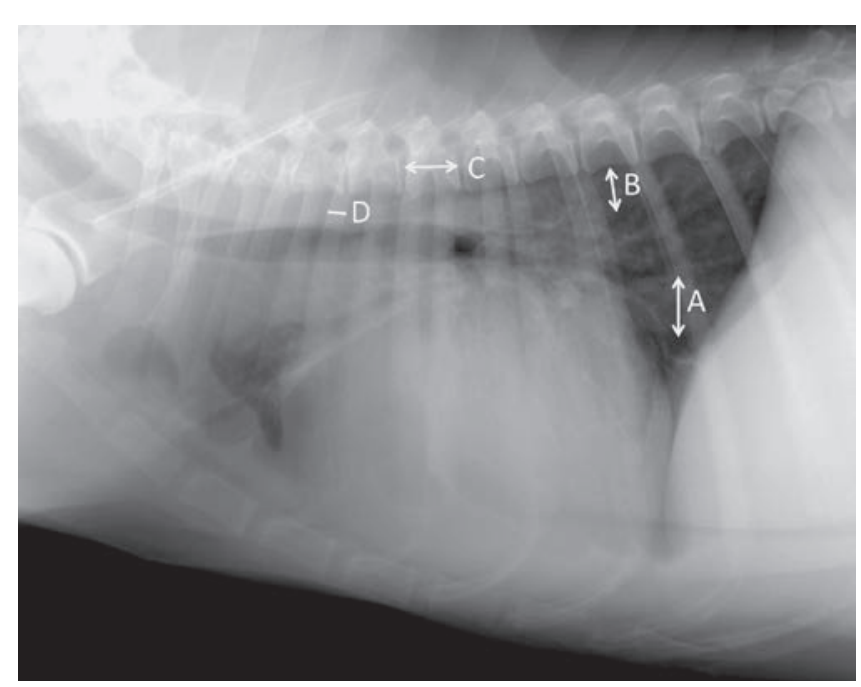

Abb. 2 Röntgenuntersuchung des Thorax im laterolateralen Strahlengang. Golden Retriever, 14 Jahre, männlich. Kardiomegalie, globoide Herzsilhouette, geringgradiger Pleuraerguss, erweiterte $\mathrm{V}$. cava caudalis (A). Vergleichsmessungen gegen die Aorta (B), den Wirbelkörper über der Bifurkation (C) und die 4. Rippe (D).

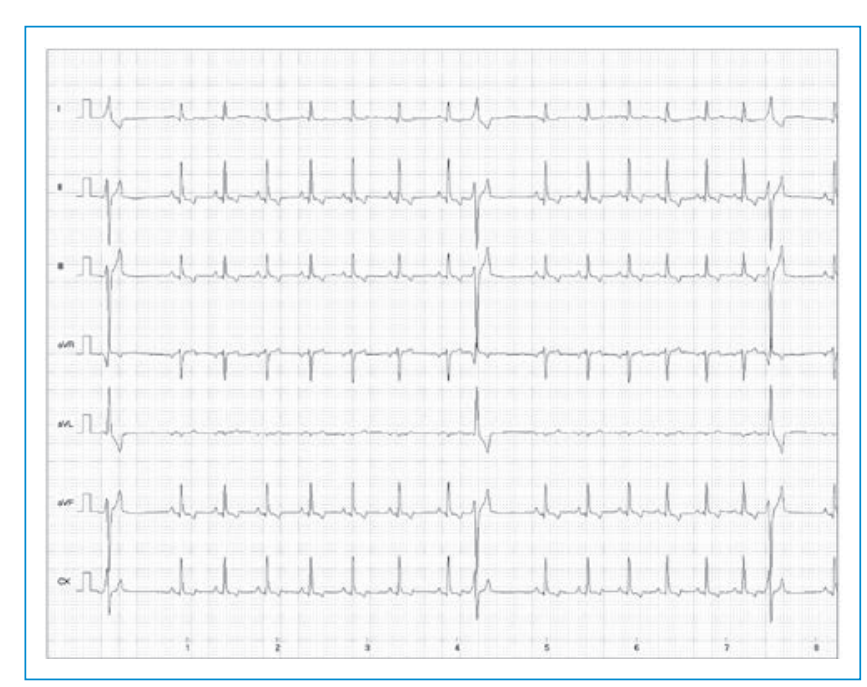

Abb. 3 Elektrokardiogramm. Deutscher Schäferhund, 9 Jahre, männlich. Ventrikuläre Extrasystolen: QRS-Komplexe verbreitert, bizarr geformt, fehlende P-Welle; zu frühes Einfallen. 


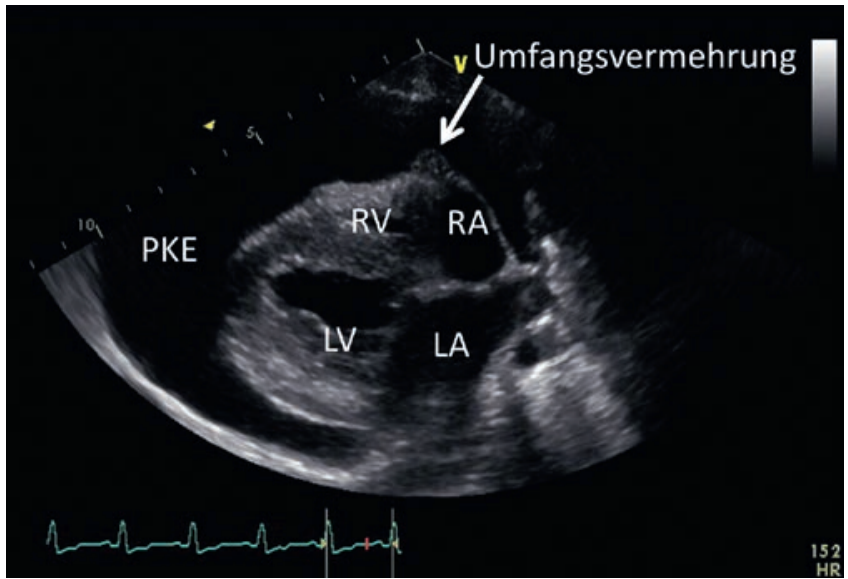

Abb. 4 Echokardiografie in rechter parasternaler Längsachse. Perikarderguss als anechogener Raum zwischen Herz und Perikard, Umfangsvermehrung am rechten Atrium (PKE: Perikarderguss; LA: linkes Atrium; LV: linker Ventrikel; RA: rechtes Atrium; RV: rechter Ventrikel).

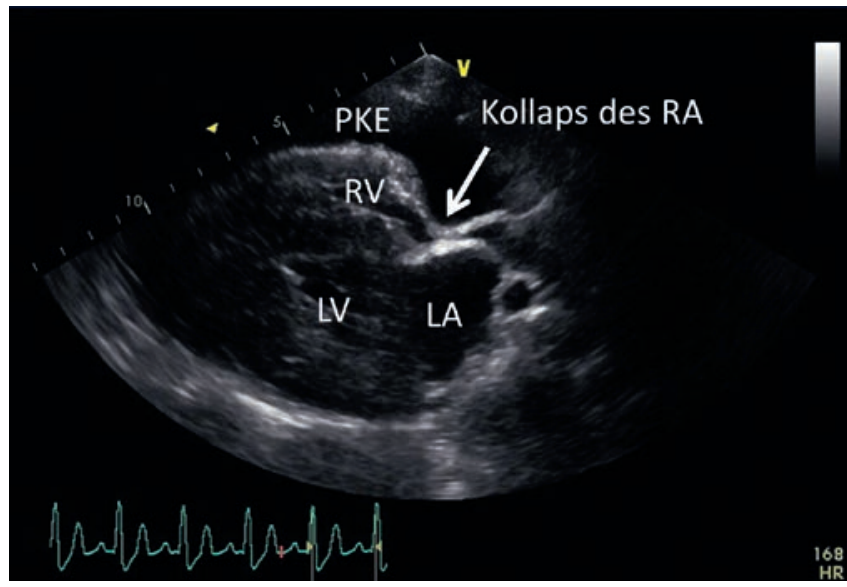

Abb. 5 Echokardiografie (rechte parasternale Längsachse): Kollaps des rechten Atriums im Zuge einer Herzbeuteltamponade (PKE: Perikarderguss; LA: linkes Atrium; LV: linker Ventrikel; RA: rechtes Atrium; RV: rechter Ventrikel). zens im Erguss und ist je nach Untersuchung bei $6-60 \%$ der Hunde zu beobachten $[4,41]$.

\section{.konkret}

Ein normaler EKG-Befund schließt einen Perikarderguss nicht aus [37].

\section{Echokardiografie}

Der Herzultraschall ist der Goldstandard für die Diagnosestellung und Einschätzung eines Perikardergusses. Für die Diagnose reichen sonografische Basiskenntnisse aus. Der Perikarderguss stellt sich als anechogener Raum zwischen Herz und Perikard dar ( Abb.4). Es können Flüssigkeitsmengen ab 10-15 ml detektiert werden [37]. Für die Einschätzung des Ausmaßes der Beeinträchtigung wird insbesondere das rechte Atrium auf einen diastolischen Kollaps und somit eine Tamponade untersucht ( Abb.5).

Ein weiteres Ziel der echokardiografischen Untersuchung ist die Identifikation potenzieller Ursachen, insbesondere von Neoplasien. Die Sensitivität für den Nachweis von Massen wird mit $82 \%$, die Spezifität mit 100\% angegeben [27,43]. Diese sollten insbesondere im Bereich des rechten Atriums und Herzohrs gesucht werden ( $\triangleright$ Abb.4). Durch den besseren Kontrast erleichtert ein bestehender Erguss das Auffinden von Umfangsvermehrungen. Es können ausgiebige, eventuell wiederholte Untersuchungen von beiden Seiten des Thorax und ein höheres echokardiografisches Geschick erforderlich sein [37]. Die Tumorsuche kann durch eine bessere Darstellung des rechten Herzens von der linken Thoraxseite aus gelingen.

Eine definitive Beurteilung einer Umfangsvermehrung hinsichtlich ihrer Dignität ist nicht möglich. Auch eine Abgrenzung gegen andere seltene Zubildungen (z.B. Thromben, Granulome, Granulationsgewebe) ist nicht sicher möglich [11,30]. Eine kavitäre Masse aus weichteildichtem Gewebe, die dem rechten Atrium entspringt, kann bei passendem Signalement (älterer Hund, große Rasse) ein Hämangiosarkom sein. Ein Tumor, der der Aorta entspringt oder diese umgibt, könnte einen Herzbasistumor darstellen [37]. Diese erste Einschätzung kann allerdings falsch sein [34].

\section{Ergussanalyse}

Eine Ergussprobe sollte in einem EDTARöhrchen stabilisiert und untersucht werden. Allerdings ist die Ergussanalyse häufig nicht diagnostisch. Unabhängig von der Ursache liegt typischerweise ein blutiger Erguss vor. Eine Studie von 1984 konnte zytologisch nicht zwischen einer Neoplasie und anderen Ursachen unterscheiden [40]. In einer Untersuchung von 273 Perikardergussproben war in 7,7\% aller Fälle eine Diagnosestellung möglich, wobei das Ergebnis bei Proben mit einem Hämatokrit unter 10\% mit 20,3\% günstiger war [6]. Vorsicht ist bei der
Diagnose eines Mesothelioms geboten, da die in Körperhöhlenergüssen vorkommenden aktivierten Mesothelzellen maligne aussehen können und so eine Fehldiagnose möglich ist [27].

Der pH-Wert des Ergusses wurde als Marker für die Malignität vorgeschlagen ([14]: erhöht; [10]: erniedrigt). Dies konnte in einer anderen Untersuchung nicht bestätigt werden [16].

\section{Sonderfall - Perikarderguss bei der Katze}

Bei der Katze stellt die kongestive Linksherzinsuffizienz die häufigste Ursache eines Perikardergusses dar [21] und erfordert somit eine andere therapeutische und diagnostische Herangehensweise.

Normalerweise handelt es sich beim Perikarderguss der Katze um einen Nebenbefund, der keine Tamponade erzeugt [44].

\section{Therapie}

Eine Perikardiozentese sollte als Notversorgung erfolgen, wenn eine Herztamponade vorliegt. Eine Stabilisierung über Infusion vor und während des Prozederes kann bereits zu einer klinischen Verbesserung führen. Wegen des ohnehin zu geringen intrakardialen Volumens und der Tatsache, dass der Erguss nicht in den Kreislauf rückresorbiert werden kann, ist Furosemid bei einem hämody- 
namisch relevanten Perikarderguss kontraindiziert.

Geringe Ergussmengen, die keine Kompression des rechten Atriums und keine Stauungssymptome bedingen, können belassen werden.

Einen Sonderfall stellt eine Vorhofruptur im Zuge einer degenerativen Mitralklappenerkrankung dar. Wenn bei einem Perikardergusspatienten ein systolisches Herzgeräusch mit Punctum maximum links apikal, eine veränderte Mitralklappe und linksatriale Dilatation sowie innerhalb des Ergusses ein Gerinnsel erkennbar sind, sollte nicht ohne eine sorgfältige Nutzen-Risiko-Abwägung punktiert werden. Hier steht die Therapie der Mitralinsuffizienz im Vordergrund.

Bei Verdacht auf eine Hypokoagulabilität sollte nur im Fall einer lebensbedrohlichen Tamponade eine Punktion erfolgen.

Die schwerwiegendsten Komplikationen im Zusammenhang mit der Perikardiozentese sind insbesondere ventrikuläre Tachykardie, Lazeration der Koronararterie, anhaltende Blutung und der plötzliche Tod. Die meisten Komplikationen treten innerhalb von 48 Stunden nach der Punktion auf $[23,29]$.

\section{.konkret}

Furosemid ist bei einem hämodynamisch relevanten Perikarderguss kontraindiziert.

\section{Adjuvante Therapie}

Eine Chemotherapie kann bei einigen Fällen von maligner Neoplasie sinnvoll sein. Bei rechtsatrialen Massen wurde nach Doxorubicin-basierter Chemotherapie ohne chirurgischen Eingriff eine mittlere Überlebenszeit von 139,5 (2302) Tagen beschrieben [17]. Die intrakavitäre Chemotherapie kann für Patienten mit Mesotheliom genutzt werden [37].

\section{Behandlung bei Rezidiv| Perikardektomie}

Beim Wiederauftreten eines tamponierenden Perikardergusses erfolgt eine erneute Perikardiozentese.

Nach spätestens 3 Punktionen sollte über eine chirurgische Therapie nachgedacht werden.
Bei einem idiopathischen Erguss oder einer peritoneoperikardialen diaphragmatischen Hernie (PPDH) kann dieser Schritt eine endgültige und bei malignen Neoplasien eine palliative Therapie darstellen [4].

Die chirurgische Therapie sollte in einer subtotalen Perikardektomie bzw. Perikardfenestration oder einer totalen Perikardektomie bestehen. Bei wiederkehrendem Erguss ist das Ziel des Eingriffs, dass sich die Flüssigkeit nicht mehr in dem begrenzt dehnbaren Herzbeutel mit geringer Resorptionskapazität, sondern im Pleuralraum mit besserer Lymphdrainage und größerer resorptiver Oberfläche ansammelt und eine Herzbeuteltamponade nicht mehr entstehen kann.

\section{Perikardektomie nach Thorakotomie}

Der traditionelle chirurgische Zugang erfolgt über eine Thorakotomie von rechts lateral oder sternal. Vorteil der Perikardektomie am offenen Thorax ist, dass das Perikard unterhalb des N. phrenicus großflächig entfernt werden kann. Zudem kann eine Identifizierung von rechtsatrialen Massen erfolgen und das rechte Herzohr, wenn dort eine isolierte Masse vorliegt, reseziert werden [1,24]. Eine Beprobung von Masse und Perikard ist möglich.

\section{Thorakoskopische Perikardfenestration} Die thorakoskopisch partielle Perikardresektion gestattet einen minimalinvasiven Zugang mit dem Nachteil, dass eine vollständige Visualisierung des rechten Atriums nicht und eine Beprobung nur eingeschränkt möglich ist und dass sich das relativ kleine geschaffene Fenster wieder verschließen kann [3, 13,24].

Eine perkutane Ballonperikardiotomie wurde ebenfalls beschrieben $[8,39]$. Dabei soll eine Öffnung im Perikard erzeugt werden, die länger als eine einfache Punktion den Abfluss gestattet. Diese Technik kommt insbesondere für Patienten mit einer Neoplasie und somit einer schlechten Langzeitprognose infrage. Der Nachteil ist, dass keine Probe für eine pathohistologische Untersuchung gewonnen werden und das Herz selbst nicht direkt dargestellt werden kann, sodass eine höhere Verletzungsgefahr für das Herz gegeben sein kann.

\section{Prognose}

Die Prognose kann sich je nach Ursache stark unterscheiden. Als negative prognostische Faktoren werden in einer Untersuchung angegeben [42]:

- Kollaps

- Abwesenheit von Aszites

- echokardiografisch erkennbare Masse

Der idiopathische Perikarderguss hat eine gute bis sehr gute Prognose. Nach einer oder mehreren Perikardiozentesen kann entweder kein Rezidiv mehr auftreten oder eine Perikardektomie erforderlich sein. In einer Studie haben noch 50\% der Patienten 1500 Tage nach Diagnosestellung gelebt [18]. Eine andere Studie hat berichtet, dass $72 \%$ noch nach 18 Monaten gelebt haben [4]. Die Prognose von Patienten mit PPDH ist ebenfalls gut.

Für Patienten mit einem Hämangiosarkom werden Überlebenszeiten von 1 $[25,42]$ bis 3 Monaten angegeben $[1,5]$, [46]. Chemotherapien (s.o. [17]) mit und ohne Perikardektomie [1] können eine etwas bessere Prognose nach sich ziehen. Nach Perikardfenestration überlebten in einer Untersuchung 8 Hunde für im Mittel 30 Tage (1-107) [3]. Chemotherapie ohne chirurgisches Vorgehen konnte in einer anderen Untersuchung eine Überlebenszeit von 139,5 Tage erzielen [17]. Vielen Hunden geht es nach einer Perikardiozentese allein sehr gut, allerdings tritt wieder ein Rezidiv auf.

Bei einem Herzbasistumor (Chemodektom, ektopes Schilddrüsenadenokarzinom) ist die Prognose wegen des langsameren Wachstums vorsichtig bis gut (mittlere Überlebenszeit ohne Perikardektomie 129 Tage, mit Perikardektomie 661 Tage [45] bzw. 42 Tage und 730 Tage [15]). Auch bei einem Mesotheliom kann nach einer Perikardektomie eine vorsichtige bis gute Prognose vorliegen. Eine Untersuchung aus dem Jahr 1998 ergab eine mittlere Überlebenszeit ab Nachweis von 13,6 Monaten, wobei $80 \%$ der Patienten 1 Jahr und 40\% 2 Jahre überlebten [12]. Eine Untersuchung von 2005 beschrieb hingegen eine mittlere Überlebenszeit von 6,5 Monaten [27].

Ein infektiöser/septischer Perikarderguss geht mit einer vorsichtigen bis guten Prognose einher und bedarf normalerweise einer Kombination aus Peri- 


\section{Durchführung einer Perikardiozentese}

\author{
Hilfspersonen und Material ( $\triangleright$ Abb.6) \\ - 1-3 Hilfspersonen \\ - Ultraschallgerät, Elektrokardiogramm \\ - Antiseptikum, Abdecktuch, sterile Handschuhe \\ - $2 \%$ iges Lidocain als Lokalanästhetikum \\ - 11er-Skalpell \\ - 14- bis 18-Gauge-Venenverweilkatheter, Heidelberger Verlängerung, \\ Dreiwegehahn, z. B. 50 ml-Perfusorspritze für Flüssigkeitsaspiration \\ - EDTA-Röhrchen für Probengewinnung; ggf. BU-Röhrchen \\ - Notfallmedikamente (insbesondere Lidocain ohne Sperrkörper)
}

\section{Vorbereitung des Patienten ( $\vee$ Abb. 7)}

- falls erforderlich Sedation (z. B. Ketamin/Diazepam); Lokalanästhesie meist ausreichend

- Infusion

- basale Blutuntersuchungen; bei Verdacht auf Koagulopathie Gerinnungsanalyse (PT/PTT)

- Positionierung in linker Seitenlage (alternativ Brustbauchlage)

- Fixierung im Idealfall durch 2 Haltepersonen, 3. Person für Anreichen und Absaugen

- EKG anschließen (nur wenn wegen Notfallcharakter Punktion unvermeidlich ohne EKG-Überwachung)

- Zugang immer von rechts (Vermeidung der großen Koronararterien links, breiterer Herz-Brustwand-Kontakt ohne Lungenparenchym)

- Ausscheren und Desinfizieren eines Feldes seitlich des Herzens im ca. 2. - 8. Interkostalraum

- Aufsuchen der Punktionsstelle

- mit Ultraschall: größten flüssigkeitsgefüllten und gut erreichbaren Raum darstellen; Punktionsstelle, z. B. durch belassene Nadel nach Lokalanästhesie markieren ( $\bullet$ Abb. 8 )

- ohne Ultraschall: Region oberhalb des palpierbaren Herzstoßes oder direkt kaudal oder unterhalb des Ellenbogens auf Höhe des kostochondralen Übergangs (ca. 5. - 6. Interkostalraum [19, 37])

- Lokalanästhesie

- cave: versehentliche Perikardpunktion kann bereits zum Entleeren des Ergusses führen: gezielt nur die Interkostalmuskulatur vorsichtig bis zur Pleura infiltrieren

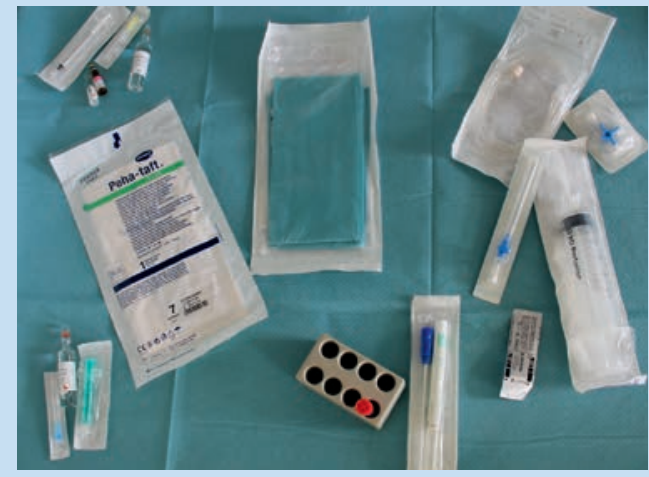

Abb. 6 Materialien für eine Perikardiozentese.

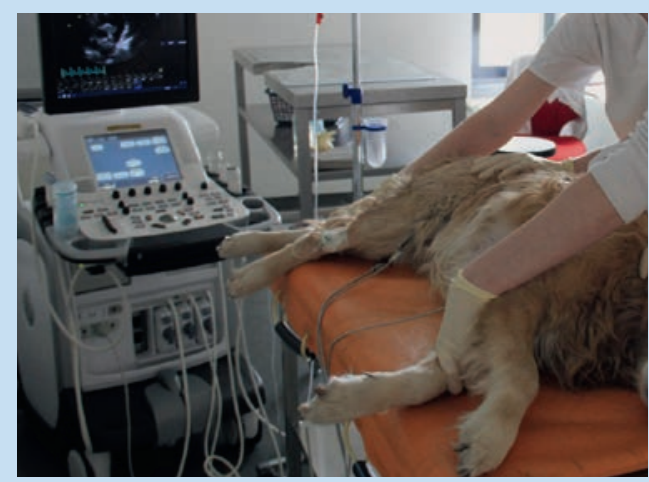

Abb. 7 Lagerung und Vorbereitung des Patienten (linke Seitenlage, EKG-Anschluss, Infusion, Schur).

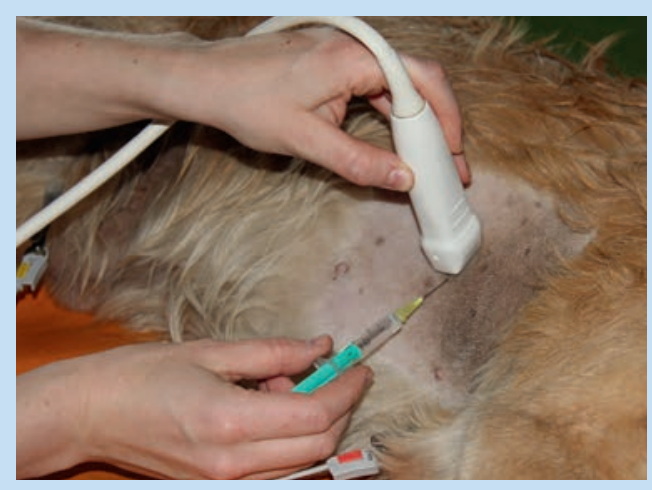

Abb. 8 Wahl der Punktionsstelle, Injektion der Lokalanästhesie (Tipp: Nadel als Markierung der Punktionsstelle in der Haut belassen). kardresektion und antibiotischer oder antifungaler Therapie [2].

Eine konstriktive Perikarditis sollte mit einer Perikardektomie und, sofern identifiziert, einer kausalen Therapie der auslösenden Erkrankung therapiert werden. Die Langzeitprognose ist allerdings vor- sichtig; 6 von 9 Hunden haben in einer Untersuchung die postoperative Zeit nicht überlebt [43].

Der sekundäre Perikarderguss bei einer kongestiven Herzinsuffizienz erreicht in aller Regel kein Ausmaß, das eine Perikardiozentese erforderlich macht. Dabei richtet sich die Prognose nach der auslösenden Erkrankung [37]. Ein Perikarderguss durch eine linksatriale Ruptur im Zuge einer degenerativen Mitralinsuffizienz hat eine schlechte Prognose [35]. 
Vorgehen ( $\vee$ Abb. 9 und $\triangleright$ Abb. 10)

- Desinfektion, sterile Abdeckung, sterile Handschuhe

- ggf. Stichinzision

- Katheter senkrecht zur Brustwand vorschieben; das Perikard ist bei vorsichtigem Vorführen als leichter Widerstand zu fühlen und gibt mit einem leichten „Popp“ nach

- Vorführen, bis Flüssigkeit im Konus erscheint (der klassische Erguss sieht wie Portwein aus)

- analog zu einem Venenkatheter nicht bei der ersten Flüssigkeit im Konus den Mandrin zurückziehen, sondern den Katheter über den Mandrin einführen und dann diesen zurückziehen

- nicht aggressiv und unkontrolliert vorgehen

- Körperhöhlenergüsse gerinnen nicht!

Gerinnselbildung: Herzpunktion oder Vorhofruptur (nur kurzer Kontakt des Blutes zu den serösen Häuten)

- insbesondere in Brustbauchlage Verwechslung mit Pleuraerguss möglich

- Verlängerung, Dreiwegehahn und Spritze ansetzen und vorsichtig aspirieren ( $\bullet$ Abb. 10)

- Stopp bei Ansaugen des Myokards (deutliches Vibrieren am Katheter, Punktat läuft nicht mehr ab) oder ventrikulären Arrhythmien ( $\triangleright$ Abb. 11): Position überprüfen, ggf. leicht zurückziehen, vorsichtig weiter evakuieren

- gerade bei geringen Ergussmengen läuft der Erguss zum Teil besser passiv ab

- bei Sistieren - falls möglich - Ultraschallkontrolle, ansonsten vorsichtig zurückziehen

- Ergussprobe in EDTA-Röhrchen geben, bei Verdacht BU-Röhrchen beschicken

\section{Überwachung nach der Perikardiozentese}

- falls möglich: kontinuierliche EKG-Überwachung wegen häufig auftretender Arrhythmien, insbesondere innerhalb der ersten 24 Stunden

- bei Schockanzeichen (Tachykardie, schlechte Pulsqualität)

Reevaluierung hinsichtlich eines Rezidivs

- medikamentelle Therapie meist nicht erforderlich (kein Vorteil

z. B. von Prednisolon, Pimobendan, Furosemid)

- Ausnahme: spezifische Indikationsstellung (z. B. Antibiose, Chemotherapie)

- Kontrolle nach z. B. 3 Wochen oder entsprechend früher bei Wiederauftreten von Symptomen

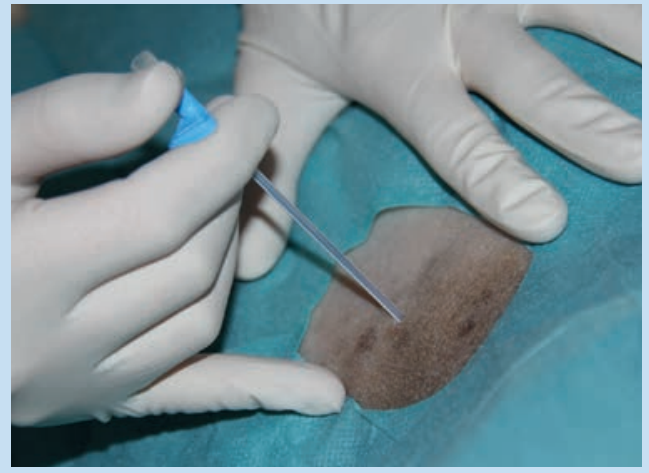

Abb. 9 Zugang mit einem großlumigen Venenzugang nach Stichinzision und antiseptischer Vorbereitung im vorgemerkten Bereich.

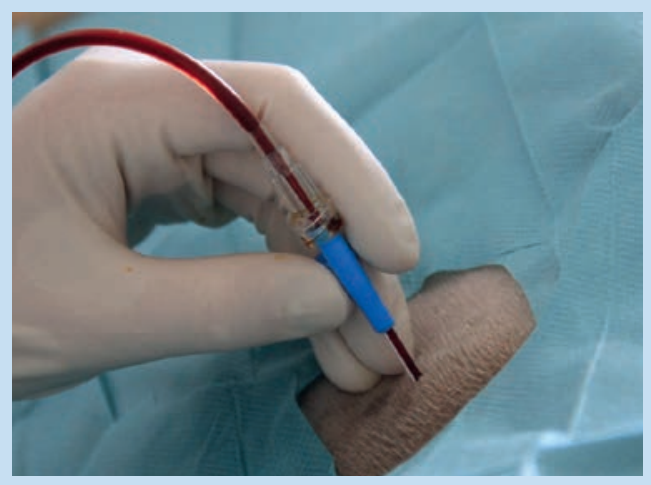

Abb. 10 Ablassen des Ergusses nach Entfernung des Mandrins.

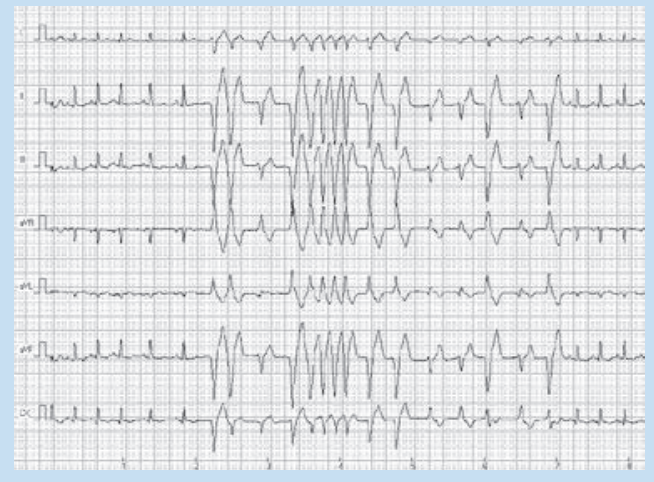

Abb. 11 Maligne ventrikuläre Arrhythmien (polymorphe Komplexe, repetitives Auftreten, R-auf-T-Phänomen): Indikation für Lidocain.

\section{Online zu finden unter}

http://dx.doi.org/10.1055/s-0034-1384417

\section{Literatur}

1 Aronsohn MG, Carpenter JL. Surgical treatment of idiopathic pericardial effusion in the dog: 25 cases (1978-1993). J Am Anim Hosp Assoc 1999; 35 (6): 521-525
2 Aronson LR, Gregory CR. Infectious pericardial effusion in five dogs. Vet Surg 1995; 24 (5): 402-407

3 Atencia S, Doyle RS, Whitley NT. Thoracoscopic pericardial window for management of pericardial effusion in 15 dogs. J Small Anim Pract 2013; 54 (11): 564-569

4 Berg R, Wingfield W. Pericardial effusion in the dog: A review of 42 cases. J Am Anim Hosp Assoc 1984; 20: 721-730
5 Brown NO, Patnaik AK, MacEwen EG. Canine hemangiosarcoma: retrospective analysis of 104 cases. J Am Vet Med Assoc 1985; 186 (1): 56-58

6 Cagle LA, Epstein SE, Owens SD et al. Diagnostic yield of cytologic analysis of pericardial effusion in dogs. J Vet Intern Med 2014; 28 (1): 66-71

7 Chun R, Kellihan HB, Henik RA et al. Comparison of plasma cardiac troponin I con- 
centrations among dogs with cardiac hemangiosarcoma, noncardiac hemangiosarcoma, other neoplasms, and pericardial effusion of nonhemangiosarcoma origin. J Am Vet Med Assoc 2010; 237 (7): 806-811

8 Cobb MA, Boswood A, Griffin GM et al. Percutaneous balloon pericardiotomy for the management of malignant pericardial effusion in two dogs. J Small Anim Pract 1996; 37 (11): 549-551

9 Côté E, Schwarz LA, Sithole F. Thoracic radiographic findings for dogs with cardiac tamponade attributable to pericardial effusion. J Am Vet Med Assoc 2013; 243 (2): 232-235

10 de Laforcade AM, Freeman LM, Rozanski EA et al. Biochemical analysis of pericardial fluid and whole blood in dogs with pericardial effusion. J Vet Intern Med 2005; 19 (6): 833-836

11 de Madron E, Prymak C, Hendricks J. Idiopathic hemorrhagic pericardial effusion with organized thrombi in the dog. J Am Vet Med Assoc 1987; 191 (3): 324-326

12 Dunning D, Monnet E, Orton EC et al. Analysis of prognostic indicators for dogs with pericardial effusion: 46 cases (19851996). J Am Vet Med Assoc 1998; 212 (8): 1279-1280

13 Dupré GP, Corlouer J, Bouvy B. Thoracoscopic pericardectomy performed without pulmonary exclusion in 9 dogs. Vet Surg 2001; 30 (1): 21-27

14 Edwards NJ. The diagnostic value of pericardial fluid $\mathrm{pH}$ determination. J Am Anim Hosp Assoc 1996; 32 (1): 63-67

15 Ehrhart N, Ehrhart EJ, Willis J et al. Analysis of factors affecting survival in dogs with aortic body tumors. Vet Surg 2002; 31 (1): 44-48

16 Fine DM, Tobias AH, Jacob KA. Use of pericardial fluid $\mathrm{pH}$ to distinguish between idiopathic and neoplastic effusions. J Vet Intern Med 2003; 17 (4): 525-529

17 Ghaffari S, Pelio DC, Lange AJ et al. A retrospective evaluation of doxorubicinbased chemotherapy for dogs with right atrial masses and pericardial effusion. J Small Anim Pract 2014; 55 (5): 254-257

18 Gibbs C, Gaskell C], Darke P et al. Idiopathic pericardial haemorrhage in dogs: a review of fourteen cases. J Small Anim Pract 1982; 23 (9): 483-500

19 Gidlewski J, Petrie JP. Therapeutic pericardiocentesis in the dog and cat. Clin Tech Small Anim Pract 2005; 20 (3): 151-155

20 Guglielmini C, Diana A, Santarelli G et al. Accuracy of radiographic vertebral heart score and sphericity index in the detection of pericardial effusion in dogs. J Am Vet Med Assoc 2012; 241 (8): 1048-1055
21 Hall DJ, Shofer F, Meier CK et al. Pericardial effusion in cats: a retrospective study of clinical findings and outcome in 146 cats. J Vet Intern Med 2007; 21 (5): 1002-1007

22 Heinritz CK, Gilson SD, Soderstrom MJ et al. Subtotal pericardectomy and epicardial excision for treatment of coccidioidomycosis-induced effusive-constrictive pericarditis in dogs: 17 cases (1999-2003). J Am Vet Med Assoc 2005; 227 (3): 435-440

23 Humm KR, Keenaghan!Clark EA, Boag AK. Adverse events associated with pericardiocentesis in dogs: 85 cases (1999-2006). J Vet Emerg Crit Care 2009; 19 (4): 352-356

24 Jackson J, Richter KP, Launer DP. Thoracoscopic partial pericardiectomy in 13 dogs. J Vet Intern Med 1999; 13 (6): 529-533

25 Kerstetter KK, Krahwinkel DJ Jr., Millis DL et al. Pericardiectomy in dogs: 22 cases (1978-1994). J Am Vet Med Assoc 1997; 211 (6): $736-740$

26 Lehmkuhl LB, Bonagura JD, Biller DS et al. Radiographic evaluation of caudal vena cava size in dogs. Vet Radiol Ultrasound 1997; 38 (2): 94-100

27 MacDonald KA, Cagney O, Magne ML. Echocardiographic and clinicopathologic characterization of pericardial effusion in dogs: 107 cases (1985-2006). J Am Vet Med Assoc 2009; 235 (12): 1456-1461

28 MacGregor JM, Rozanski EA, McCarthy RJ et al. Cholesterol-based pericardial effusion and aortic thromboembolism in a 9-year-old mixed-breed dog with hypothyroidism. J Vet Intern Med 2004; 18 (3): 354-358

29 Martin M. Pericardial disease in the dog. J Small Anim Pract 1999; 53: 381-385

30 Mathiesen DT, Lammerding J. Partial pericardectomie for idiopathic hemorrhagic pericardial effusion in the dog. J Am Anim Hosp Assoc 1985; 21: 41-47

31 Park C, Lim CY, Kim JH et al. Successful therapy of coumatetralyl rodenticide induced pericardial effusion with pericardiocentesis in a dog. Can Vet J 2011; 52 (2): 165-168

32 Parra JL, Mears EA, Borde DJ et al. Pericardial effusion and cardiac tamponade caused by intrapericardial granulation tissue in a dog. J Vet Emerg Crit Care 2009; 19 (2): 187-192

33 Petrus DJ, Henik RA. Pericardial effusion and cardiac tamponade secondary to brodifacoum toxicosis in a dog. J Am Vet Med Assoc 1999; 215 (5): 647-648

34 Rajagopalan V, Jesty SA, Craig LE et al. Comparison of presumptive echocardiographic and definitive diagnoses of cardiac tumors in dogs. J Vet Intern Med 2013; 27 (5): 1092-1096
35 Reineke EL, Burkett DE, Drobatz KJ. Left atrial rupture in dogs: 14 cases (1990-2005). J Vet Emerg Crit Care 2008; 18 (2): 158-164

36 Shaw SP, Rush JE. Canine pericardial effusion: pathophysiology and cause. Compend Contin Educ Vet 2007; 29 (7): 400-404

37 Shaw SP, Rush JE. Canine pericardial effusion: diagnosis, treatment, and prognosis. Compend Contin Educ Vet 2007; 29 (7): 405-411

38 Shaw SP, Rozanski EA, Rush JE. Cardiac troponins I and $\mathrm{T}$ in dogs with pericardial effusion. J Vet Intern Med 2004; 18 (3): 322-324

39 Shoemaker W. Pericardial tamponade. In: Grenvik A, ed. Textbook of Critical Care. Philadelphia: WB Saunders; 2000: 1097-1101

40 Sidley JA, Atkins CE, Keene BW et al. Percutaneous balloon pericardiotomy as a treatment for recurrent pericardial effusion in 6 dogs. J Vet Intern Med 2002; 16 (5): 541-546

41 Sisson D, Thomas WP, Ruehl WW et al. Diagnostic value of pericardial fluid analysis in the dog. J Am Vet Med Assoc 1984; 184 (1): $51-54$

42 Smith F, Rush J. Diagnosis and treatment of pericardial effusion. In: Kirk R, Bonagura J, eds. Kirk's Current Veterinary Therapy XIII. Philadelphia: WB Saunders; 1999: 772-777

43 Stafford Johnson M, Martin M, Binns S et al. A retrospective study of clinical findings, treatment and outcome in 143 dogs with pericardial effusion. J Small Anim Pract 2004; 45 (11): 546-552

44 Thomas WP, Reed JR, Bauer TG et al. Constrictive pericardial disease in the dog. J Am Vet Med Assoc 1984; 184 (5): 546-553

45 Tobias AH, McNiel EA. Pericardial disorders and cardiac tumors. In: Tilley LP, Smith FWK, Oyama MA, Sleeper MM, eds. Manual of Canine and Feline Cardiology. 4th ed. St. Louis, Missouri: Saunders Elsevier; 2008: 200-214

46 Vicari ED, Brown DC, Holt DE et al. Survival times of and prognostic indicators for dogs with heart base masses: 25 cases (19861999). J Am Vet Med Assoc 2001; 219 (4): 485-487

47 Ware WA, Hopper DL. Cardiac tumors in dogs: 1982-1995. J Vet Intern Med 1999; 13 (2): 95-103

\section{Dr. Katrin Baldauf}

Veterinärmedizinische Fakultät

der Universität Leipzig

Klinik für Kleintiere

An den Tierkliniken 23

04103 Leipzig 


\section{Fragebogen}

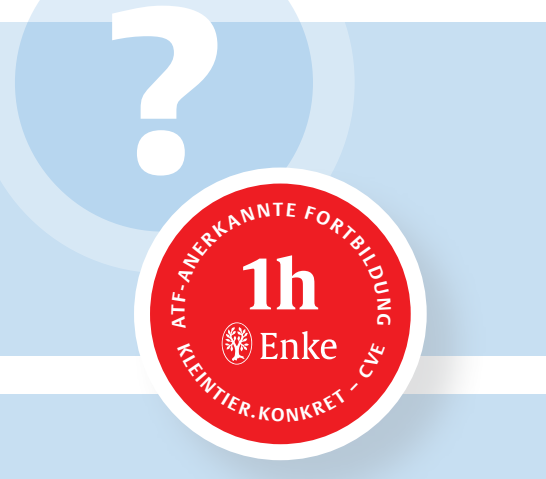

Frage 1
Welcher Schritt ist kontraindiziert, wenn ein tamponierender Perikarderguss vermutet wird?
a. Furosemidgabe
b. Echokardiografie
c. Röntgenuntersuchung
d. Infusionstherapie
e. Blutuntersuchungen

\section{Frage 2}

Welcher Befund ist in der klinischen Untersuchung bei einem Patienten mit tamponierendem Perikarderguss zu erwarten?
a. exspiratorische Dyspnoe
b. Bradykardie
c. Zyanose
d. gestaute Jugularvenen
e. Hämoptysis

\section{Frage 3}

Welche Aussage zur Echokardiografie ist richtig?

a. Echokardiografie ist der Goldstandard für die Beurteilung eines Perikardergusses.

b. Echokardiografie erlaubt den sicheren Ausschluss von Neoplasien.

c. Echokardiografie erkennt keine Flüssigkeitsvolumina unter $200 \mathrm{ml}$.

d. Echokardiografie erlaubt keine Aussage über die hämodynamische Beeinflussung.

e. Echokardiografie sollte nicht vor der Perikardiozentese erfolgen.

\section{Frage 4}

Welcher der folgenden Befunde ist stark hinweisend auf einen Perikarderguss?
a. inverser Puls
b. paradoxer Puls
c. später Puls
d. hyperkinetischer Puls
e. fehlender Puls

\section{Frage 5}

Welches diagnostische Verfahren gestattet die beste Einschätzung des Perikardergusses im Hinblick auf Ursache und hämodynamische Relevanz?
a. Blutuntersuchung
b. Elektrokardiogramm
c. Echokardiografie
d. Blutdruckmessung
e. Röntgenuntersuchung

\section{Frage 6}

Welche Ursache eines Perikardergusses sollte in einer zytologischen Ergussanalyse nachweisbar sein?
a. Hämangiosarkom
b. peritoneoperikardiale diaphragma- tische Hernie
c. bakterielle Infektion
d. Chemodektom
e. idiopathischer Perikarderguss

\section{Frage 7}

Welche ist die häufigste Ursache des Perikardergusses bei der Katze?
a. kongestive Linksherzinsuffizienz
b. Hämangiosarkom
c. Lymphom
d. Trauma
e. FIP

\section{Frage 8}

Ein 13-jähriger Rauhaardackel wird nach einem akuten Kollaps vorgestellt. Ein systolisches Herzgeräusch 4/6 mit Punctum maximum links apikal ist zusätzlich zu dem Perikarderguss auffällig. Welche Ursache ist am wahrscheinlichsten?
a. Lymphom
b. Vorhofruptur
c. Hämangiosarkom
d. histiozytäres Sarkom
e. Hypothyreose

\section{Frage 9}

Ein 9-jähriger Golden Retriever wird nach einem Kollaps vorgestellt. Auffällig ist ein tamponierender Perikarderguss mit einer kavernösen Masse im Bereich des rechten Atriums, sodass der Verdacht auf ein Hämangiosarkom gestellt wird. Welche mittlere Überlebenszeit ist nach einer Therapie per Perikardiozentese zu erwarten?
a. 1 Stunde
b. 1 Tag
c. 1 Woche
d. 1 Monat
e. $1 \mathrm{Jahr}$

\section{Frage 10}

Welcher Befund der Röntgenuntersuchung wäre klassisch für das Vorliegen eines Perikardergusses?

a. globoide Herzsilhouette, verbreiterte V. cava caudalis

b. stumpfkegelige Herzsilhouette, hypovoläme Lungenarterien

c. breitbasig aufliegende Herzsilhouette, alveoläre Lungenzeichnung

d. schmale Herzsilhouette, schmale Aorta

e. Mitralisdreieck, vaskuläre bis alveoläre Lungenzeichnung 


\section{Herzbeuteltamponade beim Hund - diagnostisches Vorgehen und Therapie}

Katrin Baldauf

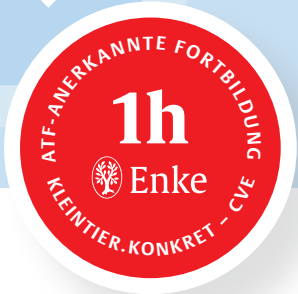

\section{A Lernerfolgskontrolle}

\section{Bitte kreuzen Sie die richtigen \\ Antworten an! Es ist jeweils nur 1 Antwort pro Frage richtig!}

\section{B Teilnehmer}

\section{Ihr Ergebnis wird vom Verlag ausgefüllt}

Sie haben von $\quad \square$ bestanden und 1 ATF-Stunde erhalten. richtig beantwortet und somit

\section{E Erklärung}

Ich versichere, dass ich die Beantwortung der

\section{Stuttgart, den}

D Teilnahmebedingungen für Abonnenten der kleintier konkret kostenlos
Fragen selbst und ohne Hilfe durchgeführt habe.

Für diese Fortbildungseinheit können Sie 1 ATF-

müssen mindestens $70 \%$ der Fragen richtig beantwortet sein.

- muss der Antwortbogen vollständig ausgefülltt sein. Unvollständig ausgefüllte Bögen können nicht berücksichtigt werden!

\section{- muss im markierten Feld* Ihre Abonnen- tennummer eingetragen oder eine kleintier konkret-CVE-Wertmarke aufgeklebt sein.}

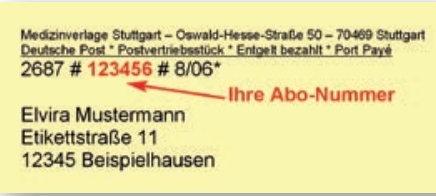

Stempel | Unterschrift

kleintier konkret-CVE-Wertmarken für NichtAbonnenten können beim Verlag zu folgenden Bedingungen erworben werden: 6erPackWertmarken, Preis 49,95€ inkl. MWSt., Artikel-Nr. 903000.

Bitte richten Sie die Bestellungen an: MVS Medizinverlage Stuttgart, KundenServiceCenter Buch, Postfach 3011 20, 70451 Stuttgart.

* Nicht-Abonnenten bitte hier kleintier konkret-CVE-Wertmarke aufkleben, Abonnenten bitte Abonnentennummer eintragen 